\title{
A COMPARISON OF SEVERAL LATTICE TOOLS FOR COMPUTATION OF ORBIT FUNCTIONS OF AN ACCELERATOR *
}

\author{
Ernest D. Courant, Scott J. Berg, and Dejan Trbojevic, BNL, Upton, New York
}

Richard Talman, Cornell University, Ithaca, New York

Al A. Garren, LBL, Berkeley, California

Abstract

The values of orbit functions for accelerator lattices as computed with accelerator design programs may differ between different programs. For a simple lattice, consisting of identical constant-gradient bending magnets, the functions (horizontal and vertical betatron tunes, dispersions, closed orbit offsets, orbit lengths, chromaticities etc.) can be evaluated analytically. This lattice was studied with the accelerator physics tools SYNCH [1], COSY INFINITY [2], MAD [3], and TEAPOT [4]. It was found that while all the programs give identical results at the central design momentum, the results differ substantially among the various lattice tools for non-zero momentum deviations. Detailed results and comparisons are presented.

\section{INTRODUCTION}

The neutrino factory or muon-collider project requires very fast muon acceleration due to a short muon lifetime. The non-scaling FFAG: fixed alternating gradient field synchrotron in recent analyses has been shown to be a promising solution [5]. The lattice design of such a synchrotron requires coverage of a very large range of momentum in the fixed magnetic field $(\delta p / p \sim \pm 40 \%)$. A simple test case was used to compare various lattice design codes. For this case the Courant-Snyder lattice functions can be calculated analytically, and the results compared to results obtained using the accelerator physics codes SYNCH, MAD, COSY, and TEAPOT over a wide momentum range $(\delta p / p<40 \%)$. We use the equations of particle motion as presented, for example, in the first lecture in the 1981 accelerator physics school [6]. In the third section, we show the results for the dependence on momentum of the lattice functions. The fourth section is a summary of the results.

\section{A SIMPLE CYCLOTRON}

\subsection{Accelerator physics Relations}

The equations of motion [2] are:

$$
\begin{aligned}
& \frac{\partial^{2} x}{\partial s^{2}}=-\frac{x}{\rho^{2}}+\frac{B_{y}-B_{0}(s)}{B \rho} \\
& \frac{\partial^{2} y}{\partial s^{2}}=-\frac{B_{x}}{B \rho}
\end{aligned}
$$

We take the magnetic field to be:

$$
\begin{aligned}
& B_{y}=B_{0}\left(1-\frac{n_{0} x}{\rho_{0}}\right)=B_{0}+G x \\
& B_{x}=B_{0} \frac{n_{0} y}{\rho_{0}} .
\end{aligned}
$$

Where $\rho_{o}$ is the central radius, $B_{0}$ the central field, and we assume the field depends linearly on the radius with gradient $G=-n_{0} B_{0} / \rho_{0}$, with no nonlinearity. The central radius is equal to $\rho_{0}=p_{0} / e B_{0}$. With a momentum offset $\delta$ defined by $\delta=\Delta p / p_{0}$, the closed orbit is a circle of radius $\rho_{0}+x_{0}$, where the magnetic field $B_{\mathrm{y}}$ equals $B_{0}+G x_{0}$; we have the equation:

$$
e\left(B_{0}+G x_{0}\right)\left(\rho_{0}+x_{0}\right)=p_{0}(1+\delta)
$$

To simplify the equation (5) we introduce a new variable $u=x_{o} / \rho_{o}$ and define $\lambda=\left(1-n_{o}\right) / 2 n_{o}$. We obtain the equation:

$$
n_{0} u^{2}-\left(1-n_{0}\right) u+\delta=0
$$

The solutions of which are:

$$
u_{1,2}=\lambda \pm \sqrt{\lambda^{2}-\frac{\delta}{n_{0}}}
$$

(The minus sign should be chosen) The two transverse equations of motion for oscillations about the circle of radius $\rho=\rho_{o}+x_{o}$ are as usual:

$$
\begin{aligned}
& \frac{\partial^{2} x}{\partial s^{2}}=-\frac{(1-n)}{\rho^{2}} x \\
& \frac{\partial^{2} y}{\partial s^{2}}=-\frac{n}{\rho^{2}} y, \text { The local field index } n \text { is: } \\
& n=n_{o} \frac{1+u}{1-n_{o} u} .
\end{aligned}
$$

The condition for stable solutions is $0<n<1$. The analytical solutions for the Courant-Snyder lattice parameters like horizontal and vertical tunes $v_{x}$ and $v_{y}$, amplitude functions $\beta_{x}$ and $\beta_{y}$, are:

$$
v_{x}=\sqrt{1-n}, v_{y}=\sqrt{n}, \beta_{x}=\frac{\rho}{\sqrt{1-n}}, \beta_{y}=\frac{\rho}{\sqrt{n}} .
$$

*Work performed under Contract Number DE-AC02-98CH10886 under the auspices of the US Department of Energy 
The horizontal and vertical chromaticies $\xi_{x}$, and $\xi_{y}$, are defined to be the derivatives of the tunes with respect to the relative momentum offset $\delta: \xi_{x}=\partial v_{x} / \partial \delta$ and $\xi_{y}=\partial v_{y} / \partial \delta$. The dispersion $D_{x}$, and the momentum compaction factor $\alpha_{\mathrm{c}}$ are: $D_{x}=\partial x_{0} / \delta=\rho /(1-n) \quad\left(\right.$ as $\left.\partial x \equiv D_{x} \delta p / p=D_{x} \delta\right)$ and $\alpha_{\mathrm{c}}=D_{x} / \rho=1 /(1-n)$.

\subsection{The test case-cyclotron 100 meters long}

To make the easiest comparison for the large off momentum offsets we used a ring with a circumference of $C_{o}=100 \mathrm{~m}$, made up of five combined-function constantgradient magnets, each $20 \mathrm{~m}$ long, with the central rigidity of $B \rho=50 \mathrm{Tm}$, and with the field index $n_{0}=0.5$. All other ring parameters like the central bending radius $\rho_{0}$, the vertical bending magnetic field $B_{\mathrm{o}}$ follow as $\rho_{o}=C_{o}$ $/ 2 \pi$ and $B_{o}=50.0 / \rho_{\mathrm{o}}$. The betatron functions for the central energy are calculated correctly in all of the codes (MAD, SYNCH, COSY, TEAPOT).

\section{RESULTS}

A few details of use of the accelerator physics codes should be noted. The COSY INFINITY code was set up to do the transfer matrix calculation with an $8^{\text {th }}$ order polynomial expansion in momentum. MAD was used in the version MAD8c as implemented on the BNL computer system. The magnets are defined as sector dipoles with gradients. TEAPOT is a thin element approximation code, so the $20 \mathrm{~m}$ long dipole was divided into 8000 pieces. The SYNCH code was used with a sector dipoles definition with gradients. The analytical results for various parameters as a function of momentum, together with results from the four different codes are presented in Figs. 1. to 7. Note that all the codes agree for $-0.08<\mathrm{d}<0.08$, but only SYNCH agrees with the analytical results over the whole range. The orbit offsets $\left(x_{o}=\rho-\rho_{o}\right)$ as a function of momentum are shown in Fig. 1 .

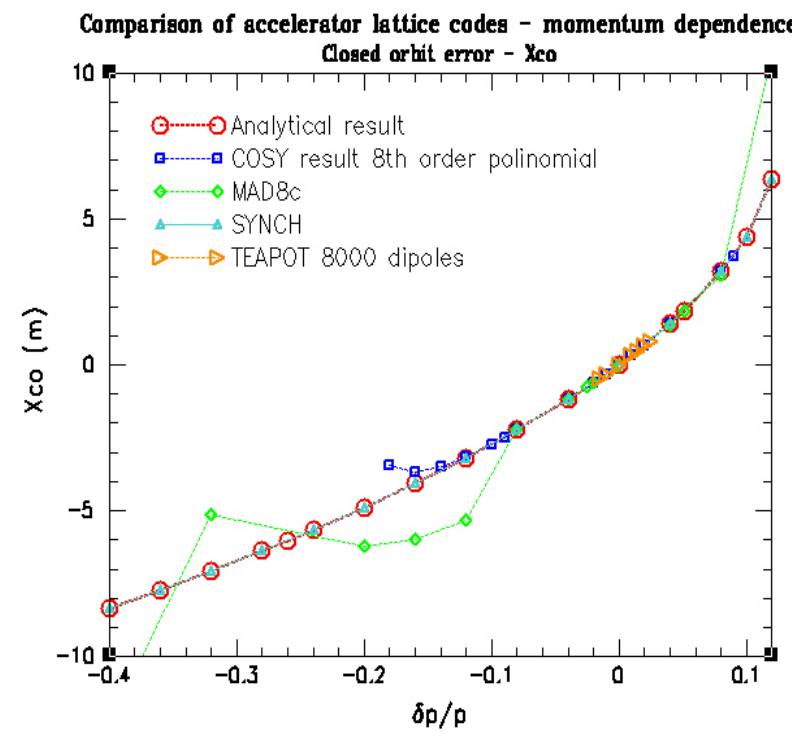

Figure 1: Orbit offset dependence on momentum.
Fig. 2 shows the change in circumference; this should of course be just $2 \pi x_{o}$, but comparing Fig. 1 and 2 we see that MAD has a discrepancy.

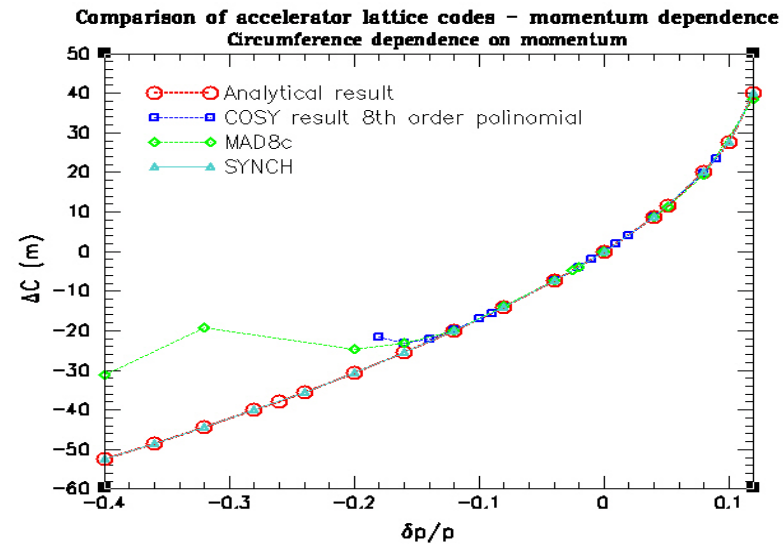

Figure 2: Change in circumference vs. momentum.

The results for the horizontal and vertical betatron are presented in Fig. 3. and Fig. 4.

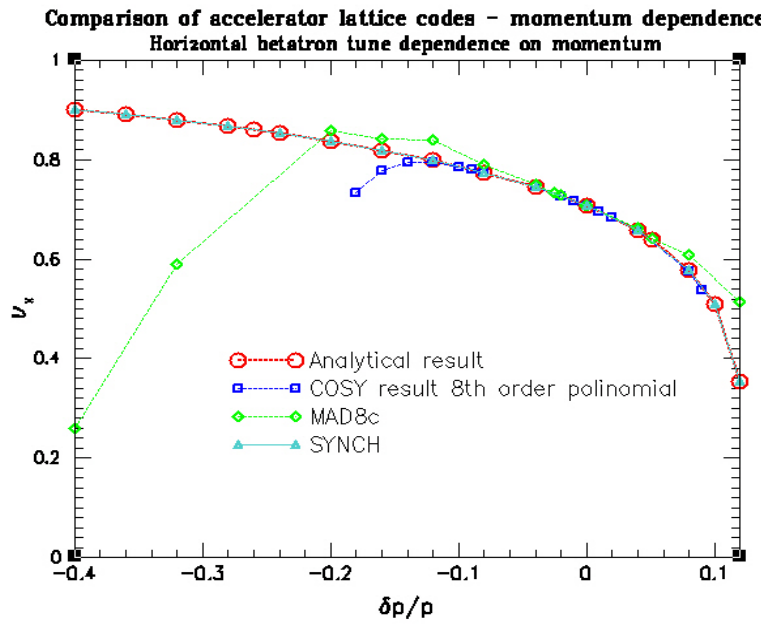

Figure 3: Horizontal betatron tune vs. momentum.

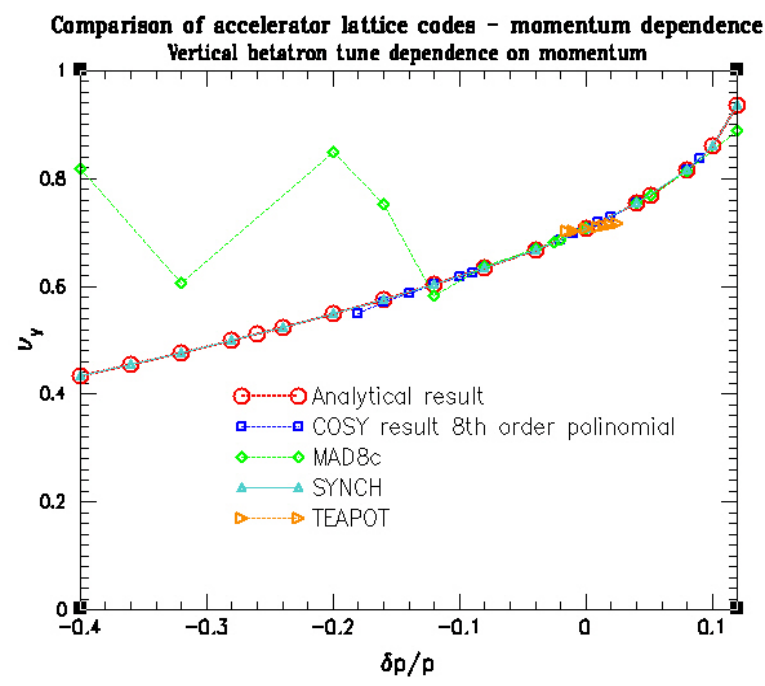

Figure 4: Vertical betatron tune vs. momentum. 
The betatron functions $\beta_{x}$ and $\beta_{y}$ vs. momentum are presented in Fig. 5 and Fig. 6, respectively.

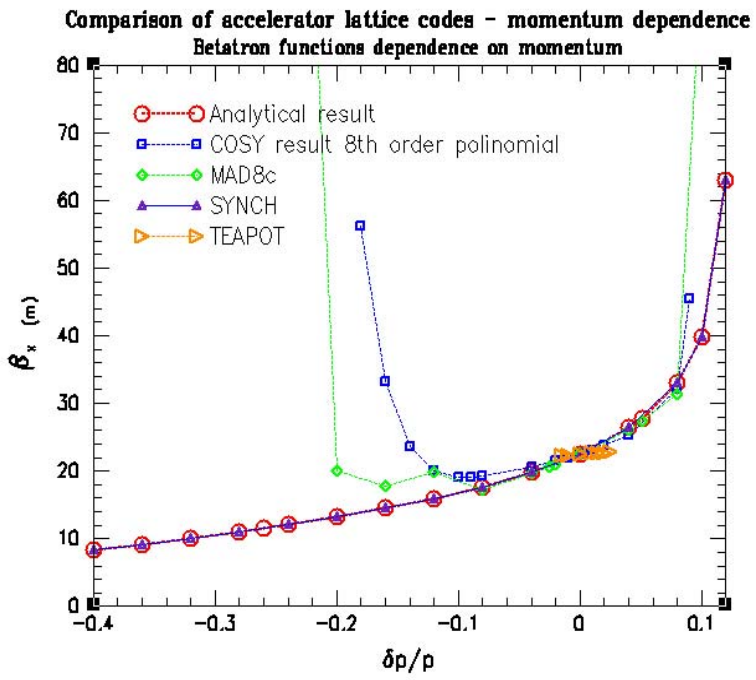

Figure 5: $\beta_{x}$ vs. momentum.

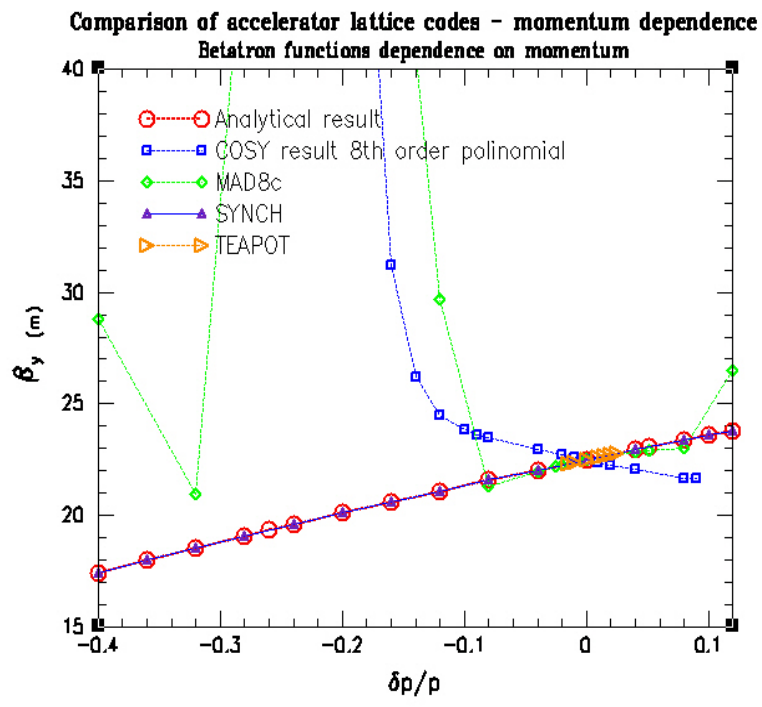

Figure 6: $\beta_{y}$ vs. momentum.

One of the most important parameters for the large momentum offsets in the lattice design is the dispersion function. The analytical prediction for the dispersion function, defined as $D_{x}=\rho /(1-n)$ in this example, together with the code results is shown in Fig. 7. The momentum compaction $\alpha_{\mathrm{c}}=D_{x} /\left(\rho_{o}+x\right)$ in this example divided by the horizontal tune should be equal to $\alpha_{c} / v_{x}=$ one. This was confirmed by SYNCH and within a limited momentum range by the other codes. The dispersion function dependence on momentum calculated analytically together with the codes results is shown in Fig. 7.

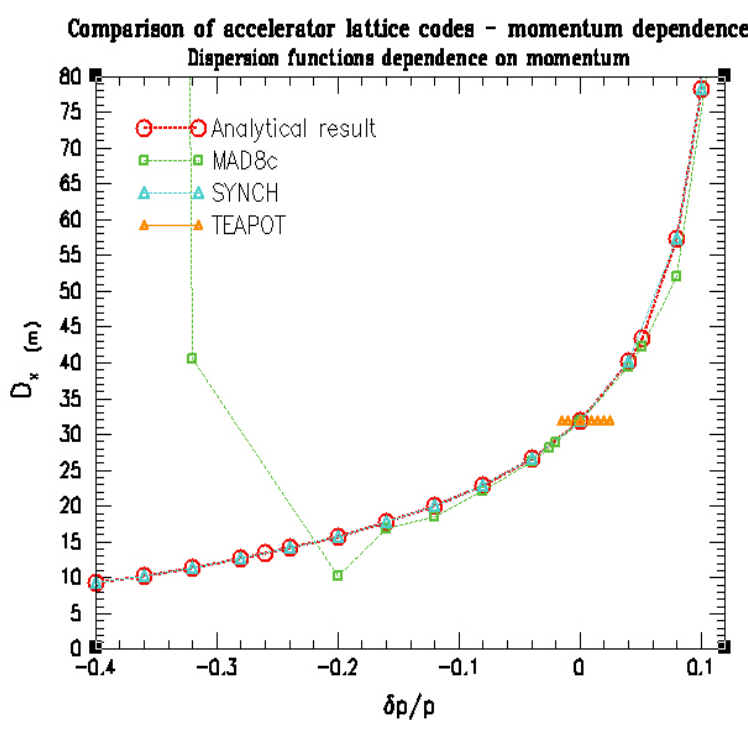

Figure 7: Dispersion function vs. momentum

\section{SUMMARY}

Large momentum offset calculations occur in some applications such as the Fixed Field Alternating Gradient synchrotron (FFAG). There is a concern with the accuracy of the existing codes for the large momentum offsets of the order of $\pm 33 \%$ and more. An easily analytically calculable example, made of five combinedfunction magnets, shows limitations in the momentum range of codes like MAD, TEAPOT, COSY INFINITY. The SYNCH code has shown perfect agreement between the analytical predictions and the code results for every lattice function examined.

\section{REFERENCES}

[1] A. Garren, A. S. Kenney, E. D. Courant, A. D. Russell, and M. J. Syphers, SYNCH-A Program for Design and Analysis of Synchrotrons and Beam Lines, User's Guide 1993.

[2] K. Makino and Martin Berz, "COSY INFINITY", Nuclear Instruments and Methods, A427, pp. 338-343, 1999, http://cosy.pa.msu.edu/.

[3] F. C. Iselin and H. Grote, MAD, "Methodical Accelerator Design", CERN/SL, 90-13 (12991).

[4] Richard Talman and Lindsay Schachinger, "Teapot: Thin Element Accelerator Program for Optics and Tracking", Particle Accelerators, 22, 35 (1991).

[5] E.D. Courant, M. Blaskiewicz, J.S. Berg, R. Palmer, D. Trbojevic, A.A. Garren, "AN UPDATE ON THE FFAG LATTICE DESIGN WITHOUT OPPOSITE BEND AND WITH DISTRIBUTED RF", at this conference, TPPG003.

[6] E. Courant, R.D. Ruth, W.T. Weng, "Introduction to Accelerator Theory", AIP Conference Proceedings, 87, American Institute of Physics, New York, 1982, pp. 4-14. 